\title{
Optimized in-flight absolute calibration for extended CMB surveys
}

\author{
B. Cappellini ${ }^{1}$, D. Maino ${ }^{1,2}$, G. Albetti ${ }^{2}$, P. Platania ${ }^{1}$, R. Paladini ${ }^{3}$, A. Mennella ${ }^{4}$, and M. Bersanelli ${ }^{1,4}$ \\ 1 Università degli Studi di Milano, via Celoria 16, 20133 Milano, Italy \\ 2 INAF-Osservatorio Astronomico, via G. B. Tiepolo 11, 34131 Trieste, Italy \\ 3 SISSA/ISAS, Astrophysics Sector, via Beirut 4, 34014 Trieste, Italy \\ ${ }^{4}$ IASF, CNR, via Bassini 15, 20133 Milano, Italy
}

Received 8 October 2002 / Accepted 4 June 2003

\begin{abstract}
Accurate measurements of the Cosmic Microwave Background (CMB) anisotropy call for high precision and reliability of the in-flight calibration. For extended surveys the CMB dipole provides an excellent calibration source at frequencies lower than $\sim 200 \mathrm{GHz}$; however poorly known foreground emissions, such as diffuse galactic components, complicate the signal and introduce a systematic error in the calibration. We show that introducing a weight function that takes into account the uncertainty in the a priori knowledge of the sky, allows us to substantially improve the calibration accuracy with respect to methods involving galactic latitude cuts. This new method is tested for PLANCK-LFI radiometers at 30 and $100 \mathrm{GHz}$. On short time scales (less than 1 day) the absolute calibration of each channel can be recovered with an overall $1-2 \%$ accuracy. We also consider the effect of CMB anisotropy itself on the calibration, and find that knowledge of the CMB pattern on large scales is needed to keep the short-time scale calibration accuracy within $1 \%$.
\end{abstract}

Key words. cosmology: cosmic microwave background - methods: data analysis

\section{Introduction}

Accurate determination of the CMB angular power spectrum can set powerful constraints on cosmological models and allows the determination of main cosmological parameters with high precision. Recently, a number of ground-based and balloon-borne experiments (De Bernardis et al. 2000; Hanany et al. 2000; Halverson et al. 2002; Pearson et al. 2003; Benoit et al. 2003) have obtained remarkable evidence for the presence of acoustic peaks in the power spectrum, extending the pioneering measurements of $C O B E$-DMR (Smoot et al. 1992) to sub-degree scales, and opening up the path to the next generation of space-based precision experiments (Bersanelli et al. 2002). In fact, it has become clear (e.g. Danese et al. 1996) that only space-based observations can provide the unique combination of environmental stability, freedom from systematic effects and avoidance of ground and atmospheric radiation needed for a high-resolution full sky survey. The WMAP satellite by NASA was launched on June 2001 and the first-year data are now available. The PLANCK satellite is an ESA mission planned to be launched in 2007.

One of the most significant systematic uncertainties affecting current CMB anisotropy experiments is the instrument calibration, typically performed by observing celestial sources with known intensity, such as planets (usually Jupiter),

Send offprint requests to: B. Cappellini,

e-mail: Benedetta.Cappellini@mi.infn.it strong radio sources (such as Cas A, Carina Nebula, Taurus A) or the $\mathrm{CMB}$ dipole. The accuracy is limited by the knowledge of the absolute flux ( $\sim 5 \%$ for planets, $\geq 8-10 \%$ for radio sources and $\sim 0.4 \%$ for CMB dipole), and also by the limited sky coverage in the case of the dipole. The WMAP and PLANCK surveys require great calibration accuracy, proportional to their instrument sensitivities.

To evaluate the accuracy actually achievable, all possible effects (both instrumental and astrophysical) impacting the calibration procedure have to be properly evaluated. In this work we focus on astrophysical effects by studying the presence of different sky emissions contaminating the main CMB dipole calibrator. In particular, we propose a method to evaluate the accuracy one can expect for absolute calibration on short time scales, by using the available information on the sky microwave emission. With this method, that can be applied to any precision CMB measurement on large sky areas, it will be possible to further improve the calibration accuracy any time more precise sky observations become available by simply reapplying the method we describe. In a forthcoming paper we will analyse instrumental effects (e.g., $1 / f$ noise, pointing uncertainties, thermal fluctuations, gain drifts) on the calibration performance.

In the present work we outline the proposed method and test the calibration technique for the PLANCK-LFI receivers.

The structure of the paper is as follows: in Sect. 2 we briefly introduce the concept of calibration; in Sect. 3 dipole contaminations (foregrounds) considered in the analysis are described. 
Section 4 shows the method to compute statistical and systematic errors in the calibration performance, and describes the different calibration techniques tested by means of simulations. Sections 5 and 6 are devoted to illustrating simulations for PLANCK-LFI and consequent results. In Sect. 7 the impact of CMB anisotropies on calibration accuracy is studied. The main conclusions and future works are discussed in Sect. 8 .

\section{Calibration concept}

Calibration is the conversion of the output signal of each detector channel (e.g. telemetry units for a space experiment) to physical units (i.e. antenna temperature ${ }^{1}$ ). In general, if the instrument response can be considered linear, then

$T_{\mathrm{A}}=G_{0} V+T_{\text {offset }}$,

where $V$ is the receiver voltage output corresponding to the sky antenna temperature $T_{\mathrm{A}}, T_{\text {offset }}$ is an instrumental offset and $G_{0}$ is the calibration factor, constant to first order. In practice, $G_{0}$ can vary due to instrumental effects, e.g. amplifiers gain or thermal instabilities.

For a differential measurement, as in the case of anisotropy experiments, the calibration is determined using pairs of sources:

$T_{2}-T_{1}=G_{0}\left(V_{2}-V_{1}\right)$

where $T_{2}-T_{1}=\Delta T_{\mathrm{A}}$ is the antenna temperature difference of sources in the sky (often a well-known bright point source and the sky background) and $V_{2}-V_{1}=\Delta V$ is the corresponding radiometer output.

In order to measure the value of cosmological parameters with great precision, calibration must be performed at $\sim 1 \%$ overall accuracy (a simple estimate of calibration requirement is shown in Appendix A).

The error in the determination of $G_{0}$ depends on the a priori uncertainty on the calibration sources temperatures, $\sigma_{\Delta T_{\mathrm{A}}}$, and on the intrinsic detector noise, $\sigma_{\Delta V}$ (Bersanelli et al. 1997):

$\frac{\sigma_{G_{0}}}{G_{0}} \simeq \frac{\sqrt{\sigma_{\Delta T_{\mathrm{A}}}^{2}+\left(G_{0} \sigma_{\Delta V}\right)^{2}}}{\Delta T_{\mathrm{A}}}$

in this expression $\sigma_{G_{0}}$ accounts for both the statistical error - intrinsic detector noise - and the systematic error - the uncertainty on the temperatures of the calibration sources -. Equation (3) shows that a better calibration is performed using higher $\Delta T_{\mathrm{A}}$ (as long as the corresponding amplitudes do not exceed the linear range of detectors).

Since Eq. (3) is an estimate of the accuracy on $G_{0}$ using only a pair of points in the sky, and since in extended surveys much more sky pixels are observed, the best value of

\footnotetext{
1 The antenna temperature $\left(T_{\mathrm{A}}\right)$ is proportional to the detector power $P$ per bandwidth $\Delta v: T_{\mathrm{A}}=P /(k \Delta v)$; it depends on both source and receiver properties. The relation between antenna and thermodynamic temperature is $T_{\mathrm{A}}=\frac{x}{\mathrm{e}^{x}-1} T$ for intensities and $\Delta T_{\mathrm{A}}=\Delta T \frac{x^{2} \mathrm{e}^{x}}{\left(\mathrm{e}^{x}-1\right)^{2}}$ for temperature fluctuations; $x=h v / k T, h$ is the Planck constant, $k$ is the Boltzmann constant and $v$ is the observing frequency.
}

the calibration constant is determined by fitting the distributions of $\Delta T_{\mathrm{A}}$ and $\Delta V$, i.e. by minimizing the one parameter $\chi^{2}$ function:

$\chi^{2}(g)=\sum_{k}\left[\frac{\Delta V_{k}-\Delta T_{k}^{\mathrm{cal}} / g}{\sigma_{\Delta V_{k}}}\right]^{2}$,

where the index $k$ refers to the pixel pairs available for calibration. In the following we indicate with $G$ the value of $g$ that minimizes $\chi^{2}(g)$, while $G_{0}$ is the true value of the calibration factor, i.e. the value we need to recover; results will be expressed in terms of $G / G_{0}$.

\subsection{The $C M B$ dipole}

An observer in motion with velocity $\beta=\boldsymbol{v} / c$ relative to the Planckian CMB field sees a dipole pattern: an angular distribution of the temperature given by

$$
\begin{aligned}
T(\vartheta) & =T_{0} \frac{\sqrt{1-\beta^{2}}}{1-\beta \cos (\vartheta)} \\
& =T_{0}+T_{0} \beta \cos (\vartheta)+O\left(\beta^{2}\right),
\end{aligned}
$$

where $T_{0}$ is the isotropic CMB temperature and $\vartheta$ is the angle between the direction of observation and the direction of $\beta$. The CMB dipole has been accurately measured by the $C O B E$ FIRAS instrument (Fixsen et al. 1996) with amplitude $\Delta T_{\mathrm{DIP}}=$ $T_{0} \beta=3.372 \pm 0.014 \mathrm{mK}$ (i.e. $\boldsymbol{v}=371 \pm 1 \mathrm{~km} \mathrm{~s}^{-1}$ ) in the direction $(l, b)=\left(264.14^{\circ} \pm 0.15,48.26^{\circ} \pm 0.15\right)$; the overall error is $\simeq 0.4 \%$.

The dipole is an ideal calibration source for CMB anisotropy experiments covering a large sky area. Its amplitude is adequate (not too strong to cause non-linear effects) and it allows a continuous calibration with no reduction of the observation time since it always enters the antenna's field of view.

\section{Foregrounds emission}

Besides the CMB dipole, different components contribute to the radio-microwave brightness of the sky. In this context, we are interested in these emissions (foregrounds) as "contaminations" of the prime calibrator, the CMB dipole. In fact, these emissions are far less precisely known than the dipole and so they represent a drawback for calibration, since they induce large errors in the determination of the $G$ factor. In our analysis we only considered diffuse components: synchrotron, free free and interstellar dust emission. We did not include additional diffuse components, such as emission from spinning dust grains (Draine \& Lazarian 1998), since a spatial and spectral full-sky template is not yet available for such components. We also did not include point sources (e.g. galactic HII regions or supernova remnants) since they fill only a very small fraction of the sky pixels. To prove their low impact we considered as an example the effect of HII regions; results will be discussed in Sect. 6.

Amplitude and spatial distribution of the considered components have been modeled (from available extended surveys) to produce synthetic sky maps at frequencies typical of 
CMB anisotropy experiments. We also estimated uncertainties on the intensity of each sky pixel for every foreground component. Maps are represented with the HEALPix ${ }^{2}$ pixelization scheme (Górski et al. 1999).

In this way we are able to produce three sky maps at each frequency of interest: a "calibrator" sky, i.e. the best knowledge of the sky we can infer today from available data:

$T^{\mathrm{cal}}(\alpha, \delta)=\sum_{i} T_{i}^{\mathrm{cal}}(\alpha, \delta)$,

an "observed" sky, which represents a deviation from $T_{\text {cal }}(\alpha, \delta)$ according to the uncertainties on the intensity of the considered components:

$T^{\mathrm{obs}}(\alpha, \delta)=\sum_{i} T_{i}^{\mathrm{obs}}(\alpha, \delta)=\sum_{i}\left(T_{i}^{\mathrm{cal}}+\sigma_{T_{i}}\right)(\alpha, \delta)$

and a full-sky map of errors $\sigma_{T}(\alpha, \delta)$ obtained adding in quadrature the error for each component:

$\sigma_{T}(\alpha, \delta)=\sqrt{\sum_{i} \sigma_{T_{i}}^{2}(\alpha, \delta)}$.

Note again that the nature of $\sigma_{T}$ is systematic.

The sum in Eqs. (5)-(7) is extended to the components considered in the simulation. The terms on the right side of Eqs. (5) and (7) are evaluated as follows.

The systematic uncertainty $(1 \sigma)$ on the dipole is estimated from the COBE uncertainty on its amplitude and direction. To be conservative we considered the worst case for calibration (see Sect. 6.1): when considering dipole, the "observed" component is a "stretched" dipole with $v=370 \mathrm{~km} \mathrm{~s}^{-1}, l=263.99^{\circ}$ and $b=48.41^{\circ}$.

To estimate synchrotron emission we used the Haslam et al. (1982) all-sky survey at $v_{0}=408 \mathrm{MHz}$, properly rescaled using a spectral indices $\beta_{i}$ map:

$T_{i}(v)=T_{i}\left(v_{0}\right)\left(\frac{v}{v_{0}}\right)^{\beta_{i}}$.

The spatial distribution of the synchrotron spectral index $\beta_{i}$ was obtained using the $408 \mathrm{MHz}, 1420 \mathrm{MHz}$ (Reich \& Reich 1986) and $2326 \mathrm{MHz}$ (Jonas et al. 1998) maps. To derive errors we took into account the $10 \%$ uncertainty of the original Haslam map plus an error on the spectral indexes determination; these errors values lie in the range $[0.1,0.8]$.

Dust emission was estimated using the $100 \mu \mathrm{m}$ intensity and 100/240 $\mu \mathrm{m}$ flux ratio map by Schlegel et al. (1998), generated from IRAS and $C O B E /$ DIRBE data ${ }^{3}$. They also provide (Finkbeiner et al. 1999) tools for dust emission extrapolation at CMB frequencies, using FIRAS data: we used their best model, a 2-components model (No. 8 in their paper) with mean dust temperatures $\left\langle T_{1}\right\rangle=9.4 \mathrm{~K}$ and $\left\langle T_{2}\right\rangle=16.2 \mathrm{~K}$, and spectral indices $\alpha_{1}=1.67$ and $\alpha_{2}=2.70$. The authors also estimate the errors on extrapolated maps to be $\sim 10 \%$.

\footnotetext{
2 http://www.eso.org/science/healpix

3 Available at http://space.gsfc.nasa.gov/astro/cobe/ dirbe_products.html
}

Diffuse free-free emission is poorly known. We only considered a free-free component spatially correlated with dust, with an intensity equal to $30 \%$ of dust at $100 \mathrm{GHz}$ (De Zotti et al. 1999), and rescaled in frequency using a power low relation:

$$
T(v)=T(100)\left(\frac{v}{100}\right)^{\alpha_{\mathrm{ff}}},
$$

where $v$ is in $\mathrm{GHz}$ and $\alpha_{\mathrm{ff}}=-2.1$, the typical Bremmstrahlung spectral index. The estimated free-free emission error is $10 \%$ from dust uncertainties plus $10 \%$ on the correlation factor.

Numerical results for the systematic uncertainty on $G$ due to the presence of poorly known foregrounds, highly depend on these estimates; anyway, the ability of the weight function technique to reduce significantly this uncertainty was successfully tested with other scenarios for the "errors" maps, so that the proposed method could also be applied in more favourable situation, e.g. taking information from the recent WMAP mission maps of the microwave emission.

\section{Determination of statistical and systematic errors on calibration performance}

To evaluate both the statistical and systematic errors on the recovered gain factor, each complete simulation is split into 2 steps:

1. We set to zero the $\sigma_{T}(\alpha, \delta)$ terms, so that the same sky map is considered: $T^{\mathrm{obs}}(\alpha, \delta)=T^{\mathrm{cal}}(\alpha, \delta)$; this is an ideal situation where we assume perfect knowledge of the observed signal. This first simulation allows us to evaluate the statistical component of the error due to instrumental noise.

2. A sky map $T^{\mathrm{cal}}(\alpha, \delta)$ different from $T^{\mathrm{obs}}(\alpha, \delta)$ is considered (Eqs. (5) and (6)). In this case both the uncertainties (astrophysical and instrumental noise) affect the simulation: the difference between the result of this second step and the first one gives the systematic component of the error.

Of course in both simulations the same noise realization has to be used.

First of all we analyse the ideal case where only the CMB dipole is considered; this allows us to evaluate the systematic error due to CMB dipole uncertainties. In this simple case, the Eqs. (5) and (6) are simplified as follows: $T^{\mathrm{obs}}=T^{\mathrm{dip}}$, $T^{\mathrm{cal}}=T_{\text {stretched }}^{\mathrm{dip}}($ see Sect. 3).

Secondly, to consider a more realistic situation, we introduce galactic emissions: we analyse the impact on $G$ of foregrounds uncertainties only, i.e. in these simulations the same dipole is considered both in the observed and in the calibrator skies, to fully analyse one type of problem at each time.

A way to handle the problem of large errors due to foreground emissions is to cut the galactic plane in the data analysis; this was for example the solution adopted by the $C O B E$ team in the analysis of DMR data (Bennett et al. 1992). This technique is limited by the presence of emission at high galactic latitudes, and is not preferable since it forces one not to consider a portion of data. 


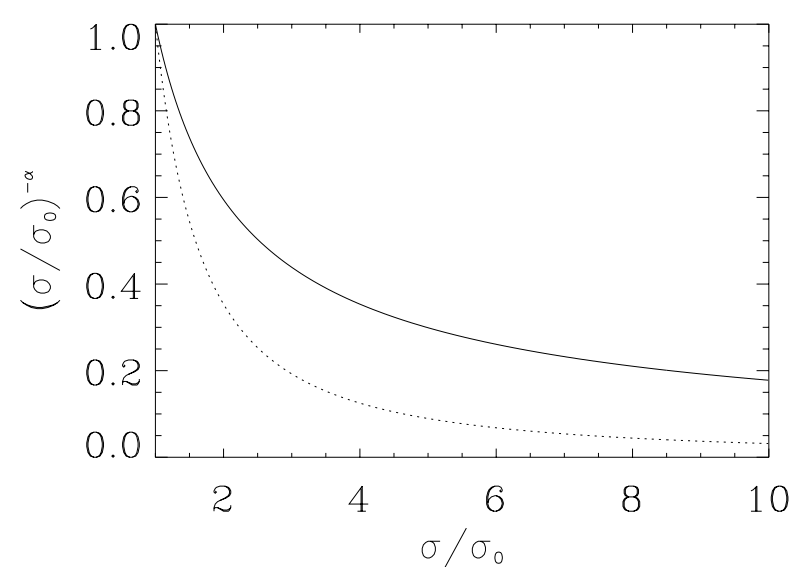

Fig. 1. Weight function for two fixed values of the $\alpha$ parameter: 0.75 (solid line) and 1.5 (dashed line).

In order to make maximum use of the whole data set, we looked for a technique able to properly weight the signal intensity $T$ of each point of the sky depending on its uncertainty $\sigma_{T}$. This can be done introducing a suitable weight function. Several functions were considered; based on our analysis we concentrated on the family of simple functions

$W\left(\sigma_{T}\right)=\frac{1}{\left(\sigma_{T} / \sigma_{0}\right)^{\alpha}}, \quad \alpha \in \mathfrak{R}$

where

$\sigma_{0}=\inf _{i}\left(\sigma_{T_{i}}\right)$.

For every value of $\alpha$ this is a decreasing function, with $\max \left(W\left(\sigma_{T}\right)\right)=W\left(\sigma_{0}\right)=1$ (Fig. 1).

When considering pixel-pairs, we use $W\left(\sigma_{\Delta T}\right)$ where

$\sigma_{\Delta T}=\sqrt{\sigma_{T_{1}}^{2}+\sigma_{T_{2}}^{2}}$,

and

$\sigma_{0}=\inf _{i}\left(\sigma_{\Delta T_{i}}\right)$

Equation (4) is then modified as follows:

$\chi_{W}^{2}(g)=\sum_{k} W\left(\sigma_{\Delta T_{k}}\right)\left[\frac{\Delta V_{k}-\Delta T_{k}^{\mathrm{cal}} / g}{\sigma_{\Delta V_{k}}}\right]^{2}$.

In this way the contribution of pixel-pairs with a poorly known temperature is reduced (at a rate controlled by $\alpha$ ), leading to a great improvement in the calibration accuracy. The value of the $\alpha$ parameter to be used is chosen in order to minimize the overall uncertainty on calibration.

For a given experiment and scanning strategy, one can study the trend of both the statistical and systematic errors, $\sigma_{\text {stat }}(\alpha)$ and $\sigma_{\text {syst }}(\alpha)$ respectively, computing the standard deviations of calculated errors over the whole mission lifetime as a function of $\alpha$. One expects the weight technique to act in opposite ways on the two types of errors: while the systematic error is a decreasing function of $\alpha$, the statistical error is an increasing one. To optimize this procedure one has to search for the minimum of the function

$f(\alpha)=\left[\sigma_{\text {syst }}^{2}(\alpha)+\sigma_{\text {stat }}^{2}(\alpha)\right]$,

yielding the optimum parameter $\alpha$.

\section{Calibration for PLANCK-LFI receivers}

PLANCK will observe CMB anisotropy and polarization with an unprecedent combination of sensitivity, sky coverage, frequency range and angular resolution. It consists of two instruments sharing the focal plane of an off-axis aplanatic 1.5 meter aperture telescope. The Low Frequency Instrument (LFI) covers the range $30-100 \mathrm{GHz}$ with four observational channels centered at 30, 44, 70 and $100 \mathrm{GHz}$. The High Frequency Instrument (HFI) will observe the sky in six channels between 100 and $857 \mathrm{GHz}$ with bolometers detectors. The wide frequency coverage will allow the separation of CMB anisotropies from non cosmological signals, thanks to their different spectral behaviour.

We applied the absolute calibration procedures described in the previous section to the case of PLANCK-LFI radiometers. A first analysis of calibration feasibility was performed in the context of the COBRAS/SAMBA ${ }^{4}$ mission study (Bersanelli et al. 1997). The HFI consortium has analysed the problem as well (Piat et al. 2002), focusing on short time scale relative calibration, and long time scale ( $\geq 1$ month) absolute calibration based on the CMB and Earth orbital motion dipoles.

During operations calibration must be performed with high accuracy and as frequently as possible, to control possible drifts on instrumental gain and offsets. The monitoring of receivers response requires a relative calibration on short time scales, while, in principle, absolute calibration can be only performed at the end of the operations and recovered through the whole mission lifetime thanks to the relative calibration. For PLANCK-LFI data, a relative calibration will be performed between the 1-hour circles thanks to the observational scanning strategy. Regarding the absolute calibration, for LFI receivers the main calibration source is the CMB dipole. Only on long time scales ( $~ 33$ months) an even better absolute calibration can be performed using the dipole modulation due to the satellite orbital motion around the Sun (which is known with high precision), as shown in Piat et al. (2002). On the other hand, in the data analysis process it will be of high interest to monitor the absolute calibration on short time scales (in particular for inter-frequency quick-look analysis comparisons) for which the "Earth-dipole" modulation cannot be used. The method described in this paper allows us to optimise the absolute calibration strategy of a full-sky mission at short time scales. In addition, this concept can be applied to balloon or ground-based experiments with limited sky coverage, for which the dipole modulation is not an effective calibrator.

To simulate LFI receiver calibration, first we created simulated data streams ${ }^{5}$ considering the PLANCK baseline scanning strategy. According to this strategy, the satellite orbits around the L2 Lagrangian point of the Earth-Sun system; the spacecraft spins at 1 r.p.m. around its spin axis which is kept along the ecliptic plane and is repointed by $2.5^{\prime}$ every hour to keep

\footnotetext{
4 COBRAS/SAMBA was the previous name of the PLANCK satel-

5 The TOD (Time Ordered Data) used in this work was generated by the PLANCK pipeline simulator of the Level-S of the DPC. Sky simulations, pointings and other data are available for PLANCK collaboration at http://planck.mpa-garching.mpg.de/SimData.
} lite. 
the anti-solar direction; the telescope field of view is at an angle of $85^{\circ}$ from the spin-axis direction.

Following the guidelines described in Sect. 3, we generate sky maps at PLANCK-LFI frequencies (30-100 GHz range); these maps are then converted into antenna temperature, and convolved with a Gaussian beam with the Full Width Half Maximum (FWHM) of LFI beams (i.e. $33^{\prime}$ at $30 \mathrm{GHz}$ and $10^{\prime}$ at $100 \mathrm{GHz}$ ) by the pipeline simulator. The code includes the main properties of the PLANCK payload (e.g. the boresight angle, the scanning strategy) and of the considered receiver (e.g. the beam location on the focal plane, its FWHM, noise properties).

The scanning strategy is such that every detector observes the same "ring" in the sky for an hour before repointing; in our work we average the 601 -minute observations of the same sky-ring, considering 1-hour data streams; we refer to these observations as "circles".

The FWHM of the antenna divides every circle $(j)$ in pixels $(i)$. The radiometer output is

$V_{i j}=\frac{T_{i j}^{\mathrm{obs}}+n_{i j}}{G_{0}}$,

where $T^{\text {obs }}$ is the observed sky, $n$ is a noise term and $G_{0}$ is the true value of the calibration constant. In the noise term we only considered white noise, i.e. a random Gaussian distribution with rms

$\delta T_{\mathrm{rms}}=\sqrt{2} \frac{T_{\mathrm{sys}}+T^{\mathrm{obs}}}{\sqrt{\Delta v \cdot \tau}}$,

where $T_{\text {sys }}$ is the system temperature of the receiver, $\Delta v$ is the bandwidth $(\Delta v / v \sim 20 \%)$ and $\tau$ is the considered integration time; the noise amplitude $\delta T_{\text {rms }}$ is quite constant during the mission. In this case, the instrumental uncertainty on output differences (see Eq. (3)) is:

$\sigma_{\Delta V} \simeq \frac{\sqrt{\delta T_{1}^{2}+\delta T_{2}^{2}}}{G_{0}}$,

where $\delta T_{1}$ and $\delta T_{2}$ are the rms values in the two observed points of the sky.

By averaging 60 rings in one circle we assume that the noise of each ring is uncorrelated with the others, which is a reasonable assumption for the low $1 / f$ noise expected from the instrument (Maino et al. 1999).

Secondly, we need to determine the pixel-pairs for calibration. If the number of pixels in a circle is $N$, then $N-1$ is the number of independent pixel-pairs; we must choose them among the $N(N-1) / 2$ possible pairs. Different criteria for choosing the $N-1$ independent pixel pairs were considered: the results obtained showed no significant changes. The simplest approach was then selected: pairs are formed with opposite pixels in a circle (Fig. 2):

1. $i \in[1, N / 2+1] \rightarrow i^{\prime}=i+N / 2-1$

2. $i \in[N / 2+2, N] \rightarrow i^{\prime}=i-N / 2-1$.

In this way every pixel is considered twice; finally the pair with the lowest signal difference is not considered, thus leaving exactly $N-1$ pairs.

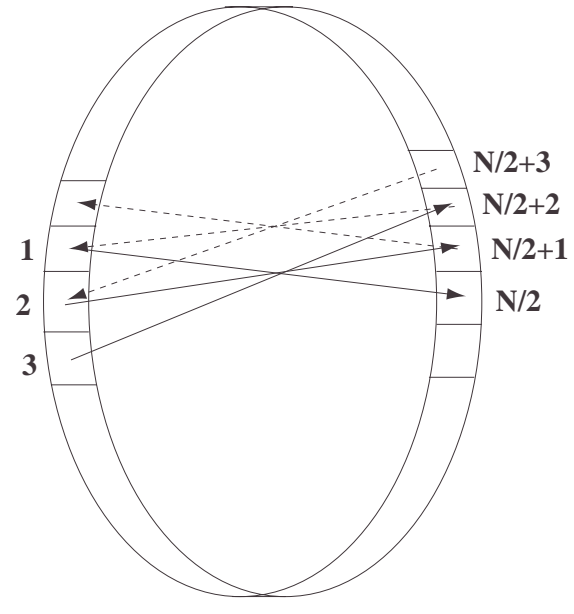

Fig. 2. Chosen pixel pairs.

The $k$ parameter in the $\chi^{2}$ function (Eq. (4)) becomes in this case $k=(m, j)$, where $m=\left(i, i^{\prime}\right)$ refers to pixel-pairs previously defined and $j$ refers to the considered circle.

In our simulations the $G$ factor is computed over two different time-scales: 1 hour and 1 day. Both are "natural" time scales for PLANCK: the first defines the scan "circles" while the second is the time scale of Earth-satellite communications. Thus, the sum in Eq. (4) is extended to the $N-1$ independent pixel pairs of one circle in the first case (fixed $j$ value), while is extended to the $(N-1) \cdot 24$ circles covered in one day in the second case.

\section{Simulation results}

We present the analysis carried out at 30 and $100 \mathrm{GHz}$. The first is the channel where foreground emission is the strongest while the second one has the highest noise level per single radiometer (for $30 \mathrm{GHz}$ radiometers $T_{\text {sys }} \simeq 10 \mathrm{~K}$, for $100 \mathrm{GHz}$ $T_{\text {sys }} \simeq 45 \mathrm{~K}$ ): they represent the worst case for systematic and statistical error behaviour respectively. The other LFI frequencies are in intermediate situations.

We applied the 2-step procedures described in Sect. 4; as already noted, the same noise realization has to be used for both steps. In the case of LFI radiometers, since the noise rms value has a slight dependence on the observed sky temperature (see Eq. (17)), considering the same realization for both simulation introduces an error of a few \%o on signal terms (Eq. (16)).

Similar results hold for all the radiometers at the same frequency. At $30 \mathrm{GHz}$ we have considered the radiometer LFI-28 with the beam position $\left(\theta_{\mathrm{B}}=5.26^{\circ}, \phi_{\mathrm{B}}=52.9^{\circ}\right)$ in the focal plane and angular resolution of $33^{\prime}$ that divides every observed circle in 1950 pixels $^{6}$; at $100 \mathrm{GHz}$ we used the radiometer LFI-01 with the beam position $\left(\theta_{\mathrm{B}}=2.93^{\circ}, \phi_{\mathrm{B}}=0.0^{\circ}\right)$ and the angular resolution of $10^{\prime}$ that divides every observed circle in 6498 pixels $^{6}$.

\footnotetext{
${ }^{6}$ Note that the dimension of a pixel is equal to FWHM/3.
} 

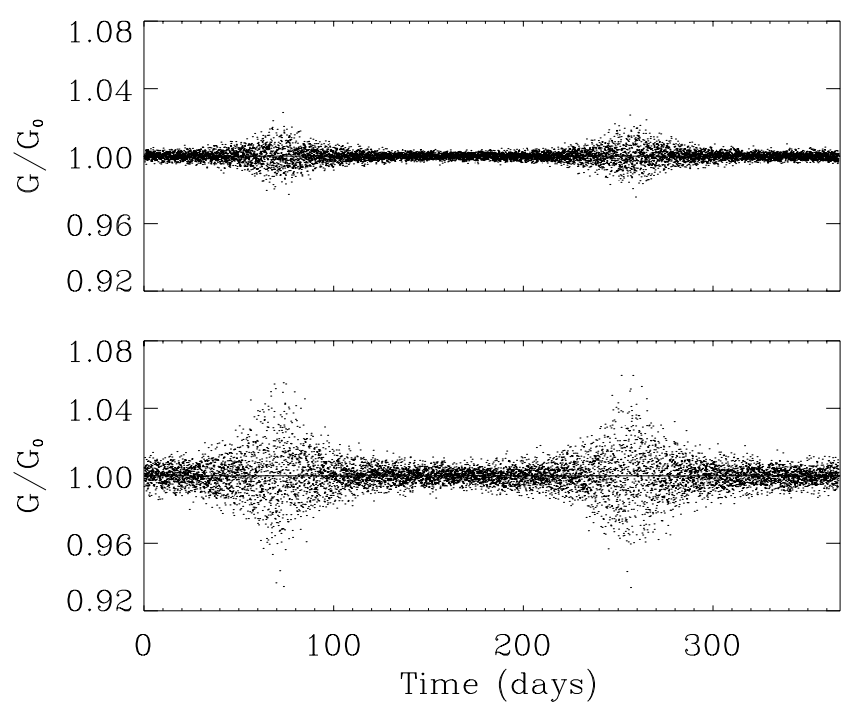

Fig. 3. Statistical error on the $G$ value calculated every hour at $30 \mathrm{GHz}$ (upper panel) and $100 \mathrm{GHz}$ (lower panel).

\subsection{CMB dipole}

As described in Sect. 4, we start by considering the CMB dipole only. This allows us to evaluate the systematic error due to CMB dipole uncertainties, and also provides a consistency test of our code.

Results on the 1-hour time scale are shown in Fig. 3 (statistical errors) and Fig. 4 (systematic errors); on such short time scales the statistical and the systematic errors are of the same order, $\sim$ few $\%$. The typical trend of the statistical error is the effect of different $\Delta T_{\text {dip }}$ values $\left(\sigma_{G_{0}} \propto \Delta T_{\mathrm{A}}^{-1}\right.$, cf. Eq. (3)) observed in the scan circles over the different periods of the mission time, due to the changing geometry of the field of view with respect to the dipole direction. The correlation is clear looking at Fig. 6, where the largest temperature difference of every circle is plotted. The shape of the systematic error depends on the choice of $\boldsymbol{v}, l$ and $b$ in the stretched dipole. In Fig. 4 (lower panel) the effect on $G$ when changing these parameters one at a time is shown. To be conservative we chose a combination (see Sect. 3) of these parameters that maximize the dipole systematic error (Fig. 4, upper panel).

As expected, the amplitude of the statistical and systematic errors are consistent with the estimate of detector sensitivities on the considered time scale $\left(\sim 0.2 \mathrm{mk} \mathrm{Hz}^{-1 / 2}\right.$ at $30 \mathrm{GHz}$ and $\sim 0.6 \mathrm{mk} \mathrm{Hz}^{-1 / 2}$ at $100 \mathrm{GHz}$ ) and with the COBE-FIRAS uncertainties on dipole measurements.

If calibration is performed every 24 hours, the statistical error (Fig. 5) shows the same trend over time but with much lower amplitude (cf. Eq. (17)); the systematic error is time independent and indeed results over different time scales perfectly overlap (results with 24 hours as time-scale are thus not shown). These time-trends of statistical and systematic errors always apply for a given calibration technique if the only difference is the time scale chosen to recover the gain factor.
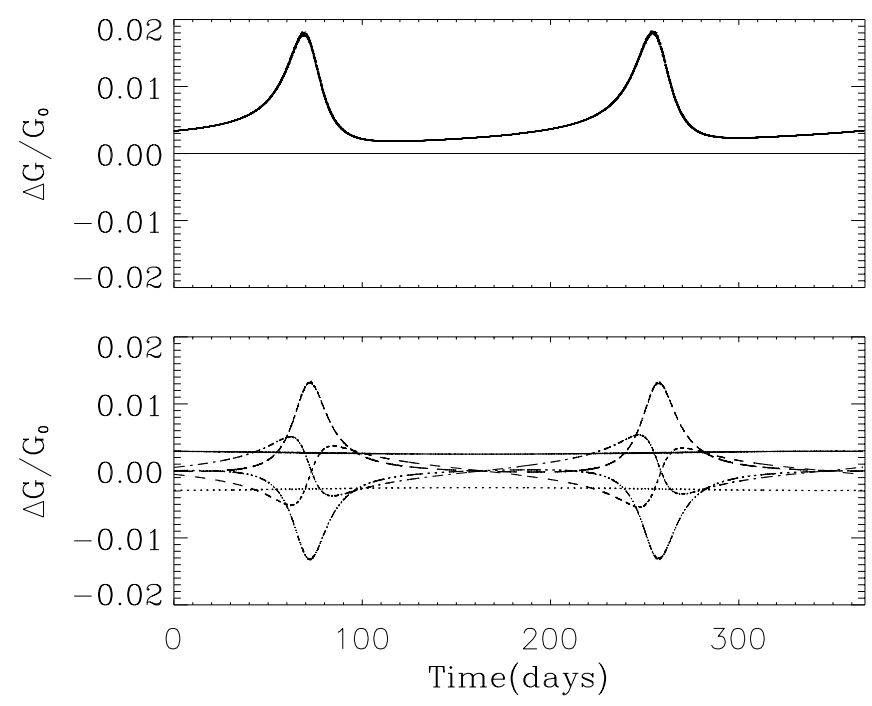

Fig. 4. Systematic error due to CMB dipole uncertainty (from the COBE measure) on the $G$ value calculated every hour, at $30 \mathrm{GHz}$ and $100 \mathrm{GHZ}$ (upper panel, plots almost perfectly overlap). In the lower panel, "partial" systematic errors are shown (see text for more details); solid line: $\boldsymbol{v}=370 \mathrm{~km} \mathrm{~s}^{-1}$, dotted line: $\boldsymbol{v}=372 \mathrm{~km} \mathrm{~s}^{-1}$, dashed line: $l=264.29^{\circ}$, dotted-dashed line: $l=263.99^{\circ}$, double dotted-dashed line: $b=48.11^{\circ}$, long dashed line: $b=48.41^{\circ}$.
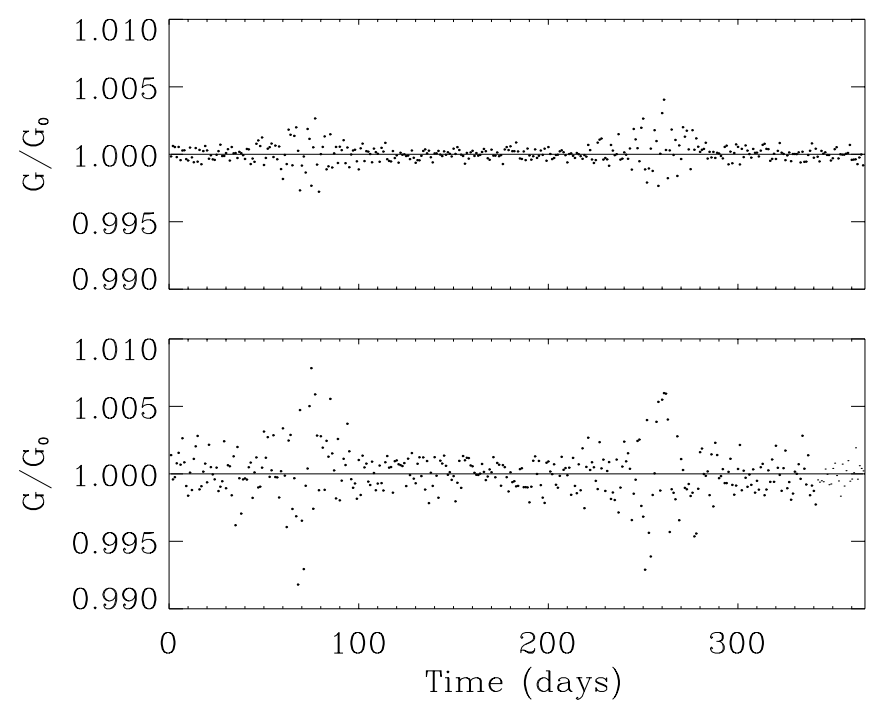

Fig. 5. Statistical error on the $G$ value calculated every 24 hours at $30 \mathrm{GHz}$ (upper panel) and $100 \mathrm{GHz}$ (lower panel).

\subsection{Foregrounds}

A more realistic situation is obtained introducing galactic emissions. Histograms for the "error"/"observed" maps of foregrounds (Sect. 3) at the considered frequencies are plotted in Fig. 7. In both cases, for less than $0.4 \%$ of the sky pixels this value is greater than 1 , while isolated pixels have very high "error"/“"observed"values.

Figure 8 shows the systematic error induced by uncertainties on foregrounds components. At both frequencies the errors are much larger than required (see Appendix A) and, as expected, the situation is far more problematic at $30 \mathrm{GHz}$. 


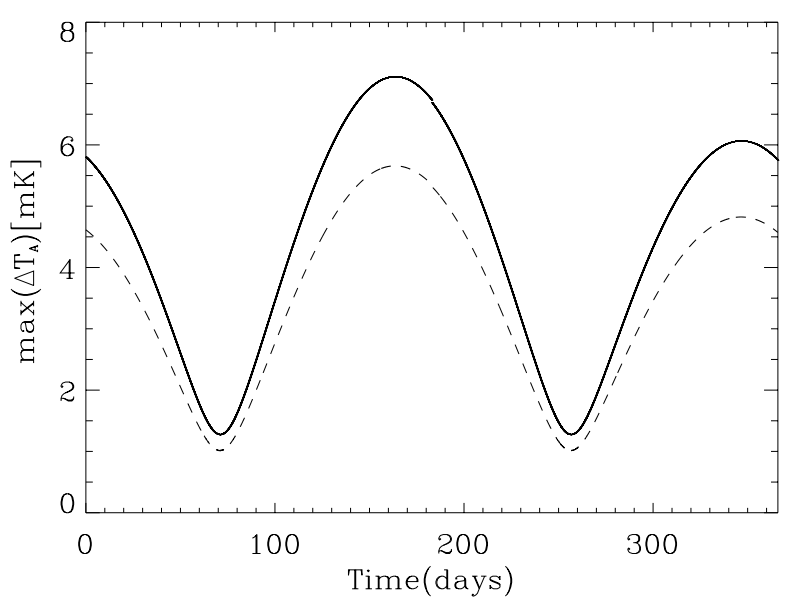

Fig. 6. Higher $\Delta T$ available for every hour-calibration at $30 \mathrm{GHz}$ (solid line) and $100 \mathrm{GHz}$ (dashed line).
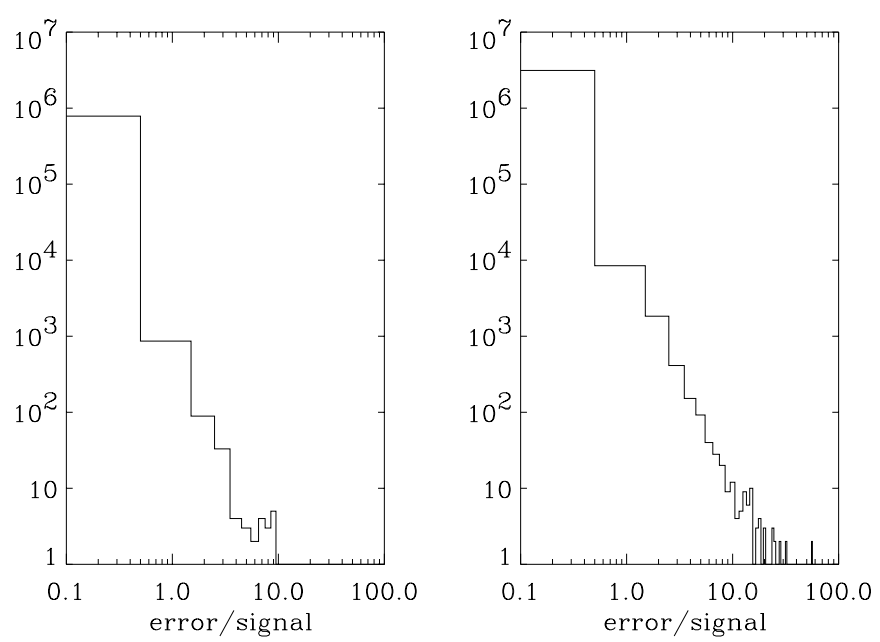

Fig. 7. Histograms for the "error"/"observed" maps of foregrounds at 30 (left panel) and 100 (right panel) GHz.

The presence of spikes in Fig. 8 means that very large errors are concentrated in limited sets of scan circles. In the following we give an explanation of the presence of such spikes.

As already mentioned in Sect. 3, these simulations only consider diffuse emission components. Anyway we investigated the impact of point sources considering galactic HII regions; we used a recent compilation of 1442 sources (Paladini et al. 2003): they affect less than $0.5 \%$ of the sky (Burigana \& Paladini 2003), contaminating $\sim 7 \%$ of scan circles in very few pixels. To be conservative, their emission was only included in the $T^{\mathrm{obs}}(\alpha, \delta)$ map: we thus calibrated assuming no information at all on their presence. Simulations show there is no impact on the statistical error, while the systematic error is increased (only in the contaminated scan circles): in practice other spikes similar to those present in Fig. 8 appear in the $G$-plot. Furthermore we run the calibration procedure excluding in the $\chi^{2}$ function (Eq. (4)) the pixels with known HII regions; again the statistical error is not affected, but we found that most of the spikes in Fig. 8 disappear. This result can be explained in a simple way: spikes in the $G$ plots are due to the largest values in our estimated error map, which
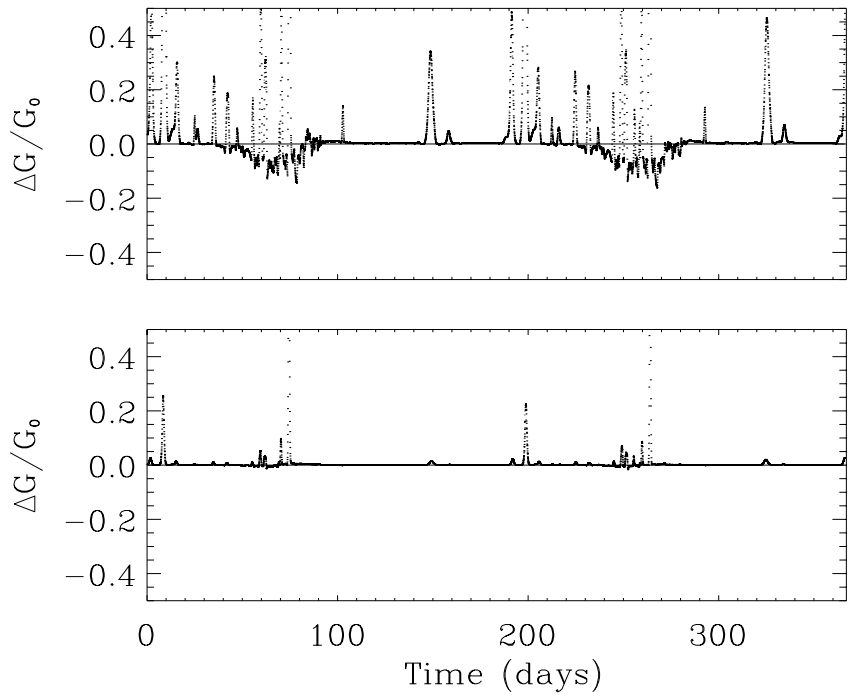

Fig. 8. Systematic error due to galaxy uncertainty on the $G$ value calculated every hour, at $30 \mathrm{GHz}$ (upper panel) and $100 \mathrm{GHz}$ (lower panel).

correspond - not a surprise - to point source pixels, and to sources not completely removed in the maps of the the dust component.

These tests show that point sources do not interfere significantly with the proposed calibration technique, as long as they can be removed with the aid of source catalogues.

On the other hand strong point sources, such as brightest HII regions and planets, can be very useful as a supplementary way to monitor the stability of the gain factor (relative calibration), since they are clearly recognizable in the received signal given their high intensity. Indeed, relative calibration only requires stable bright sources, and not a precise knowledge of their absolute intensity. When possible, relative calibration on these sources can be performed over different time scales, such as:

- less than 1 hour, since they are observed by a given detector every minute with a high signal to noise ratio;

- six months, i.e. the time needed for a given detector to come back to the same portion of the sky.

\subsubsection{Galactic cuts calibration technique}

As a further step, we simulated the calibration technique with a $\pm 20^{\circ}$ galactic cut; higher galactic cuts do not permit calibration over long periods during the mission, due to an excessive decrease in the number of avilable pixel-pairs.

We only show results with a 1-hour integration time, in Figs. 9 and 10.

As expected, the reduction in the data-set increases the statistical error (Fig. 9, compare to Fig. 3); on the other hand the systematic error is highly reduced as can be seen comparing Fig. 10 to Fig. 8. Anyway this technique is limited by the presence of residual emission at high galactic latitudes; in Fig. 10 we can see the effect of the emission of Magellanic Clouds that are at $-30^{\circ}$ of galactic latitude. At $30 \mathrm{GHz}$ their signals uncertainty produces an error of $\sim 15 \%$ on $G$. 

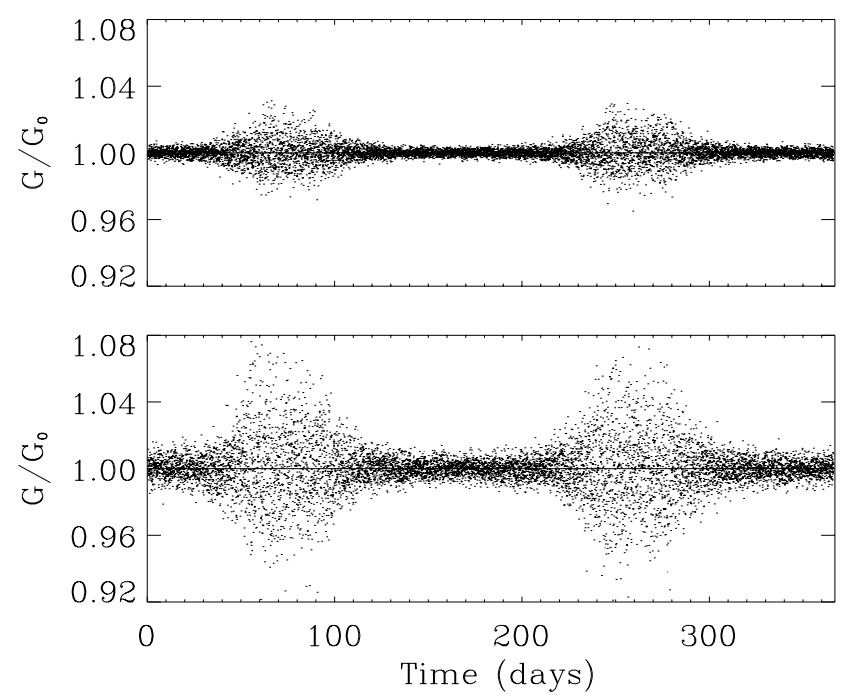

Fig. 9. Statistical error on the $G$ value calculated every hour, with a galactic cut of $20^{\circ}$, at $30 \mathrm{GHz}$ (upper panel) and $100 \mathrm{GHz}$ (lower panel).
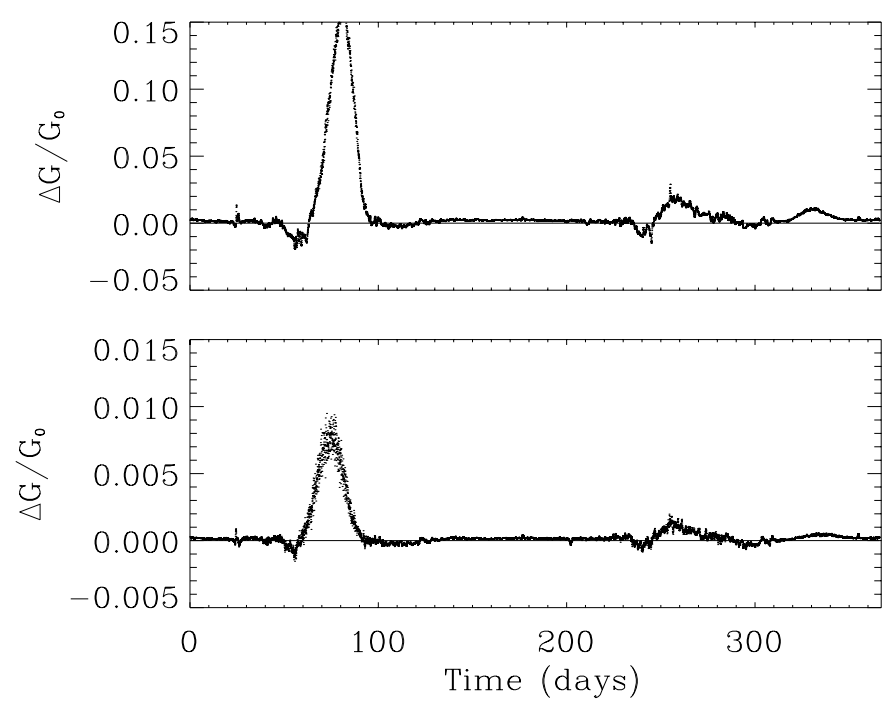

Fig. 10. Systematic error on the $G$ value calculated every hour, with a galactic cut of $20^{\circ}$, at $30 \mathrm{GHz}$ (upper panel) and $100 \mathrm{GHz}$ (lower panel). The spike in the results is the effect of Magellanic Clouds emission at high galactic latitudes.

\subsubsection{The "weight function" calibration technique}

The "weight function" technique allows us to improve the calibration accuracy. Results on the optimization of $\alpha$ are shown in Fig. 11. The optimum choice of $\alpha$ depends on both frequency and integration time. The frequency dependence is obvious, since foreground emission and noise levels are different at the various frequencies. The integration time dependence comes from the fact that the systematic error is independent of time, while white noise scales as $1 / \sqrt{\tau}$. The $\alpha$ step in simulations is 0.125 at both frequencies. On the 1-hour time scale, we find that the best result is with $\alpha=1.5$ at $30 \mathrm{GHz}$ and $\alpha=0.625$ at $100 \mathrm{GHz}$; on a 24-hour time scale the best result is with $\alpha=2.875$ at $30 \mathrm{GHz}$ and $\alpha=0.75$ at $100 \mathrm{GHz}$.
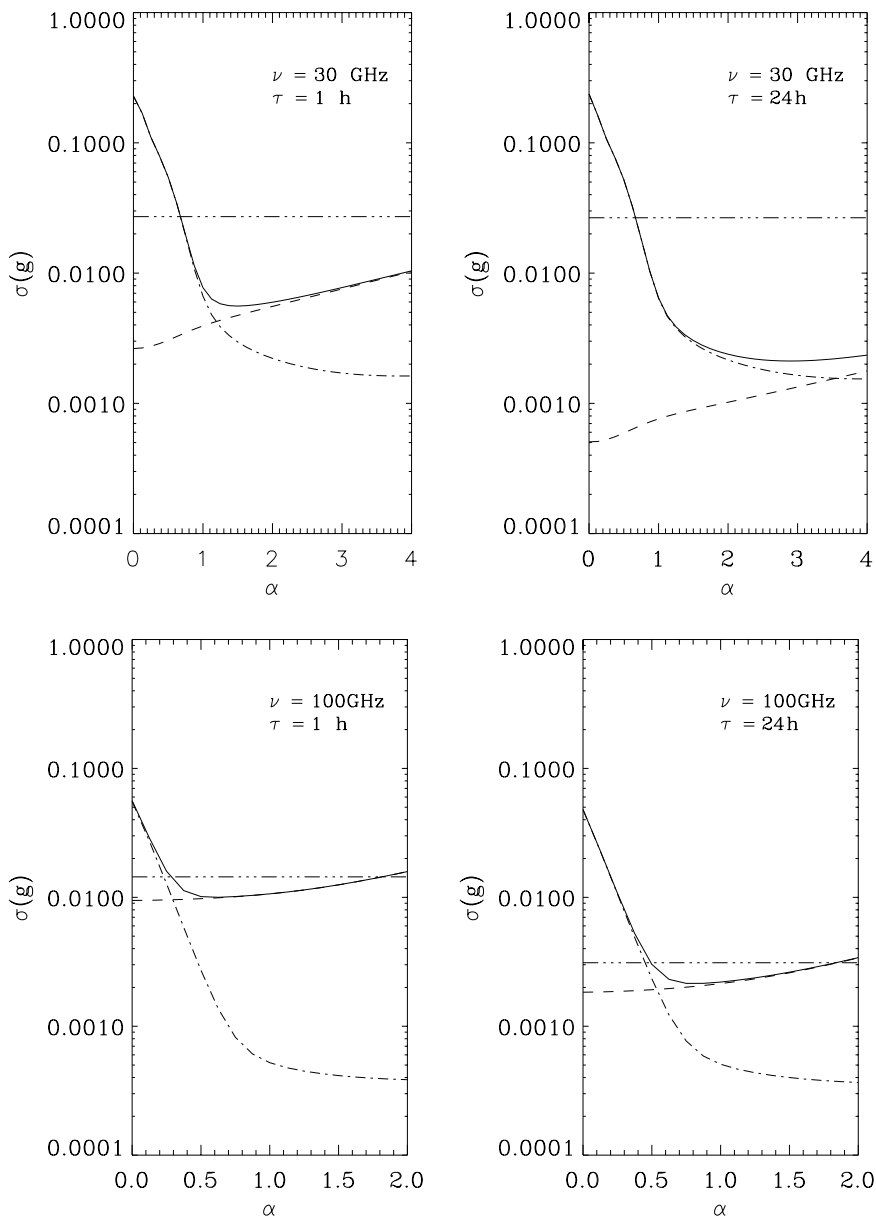

Fig. 11. Systematic (dot-dashed line) and statistical (dashed line) error behaviour as a function of the parameter $\alpha$ (defined in Eq. (10)). The best $\alpha$ value is the one minimizing $f(\alpha)$ (solid line, see Eq. (15)). The horizontal line is the total error for the $20^{\circ}$ galactic cut technique. Panels on the left correspond to a 1 hour timescale calibration; those on the right to 24 hours. Upper panels: $30 \mathrm{GHz}$ channel; lower panels: $100 \mathrm{GHz}$ channel.

Results for systematic errors are shown in Figs. 12 and 13. Considering an integration time of 1 day, one can achieve $\sigma_{\text {syst }} \leq 1 \%$ for the whole mission time at $30 \mathrm{GHz}$ (Fig. 13, upper panel) and $\sigma_{\text {syst }}$ is $\leq 0.3 \%$ pratically for $100 \%$ of the mission time at $100 \mathrm{GHz}$ (Fig. 13, lower panel). These results are in line with the requirements indicated in Appendix A.

\section{CMB anisotropies}

Finally we considered the impact of the presence of CMB intrinsic anisotropies on the calibration accuracy. Anisotropy maps were simulated with the SYNFAST routine of the HEALPix package, given a Standard Cold Dark Matter CMB power spectrum. The spectrum was obtained through the CMBFAST code $^{7}$. We used the best calibration parameters found in our foregrounds analysis.

\footnotetext{
${ }^{7}$ http://physics.nyu.edu/matiasz/CMBFAST/cmbfast.html
} 

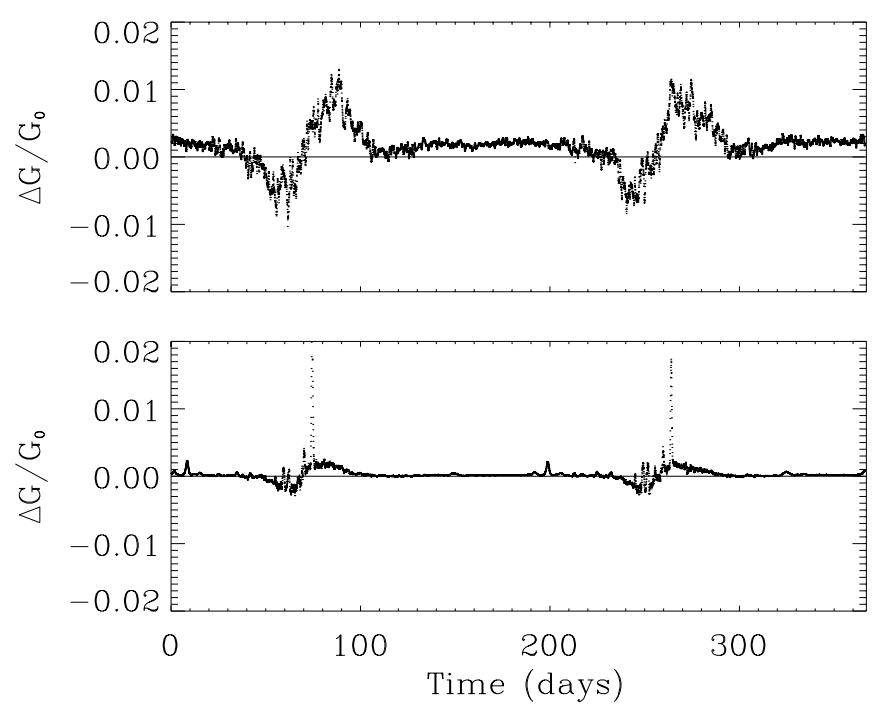

Fig. 12. Systematic error on $G$ calculated every hour, with $\alpha=1.5$ at $30 \mathrm{GHz}$ (upper panel) and with $\alpha=0.625$ at $100 \mathrm{GHz}$ (lower panel).
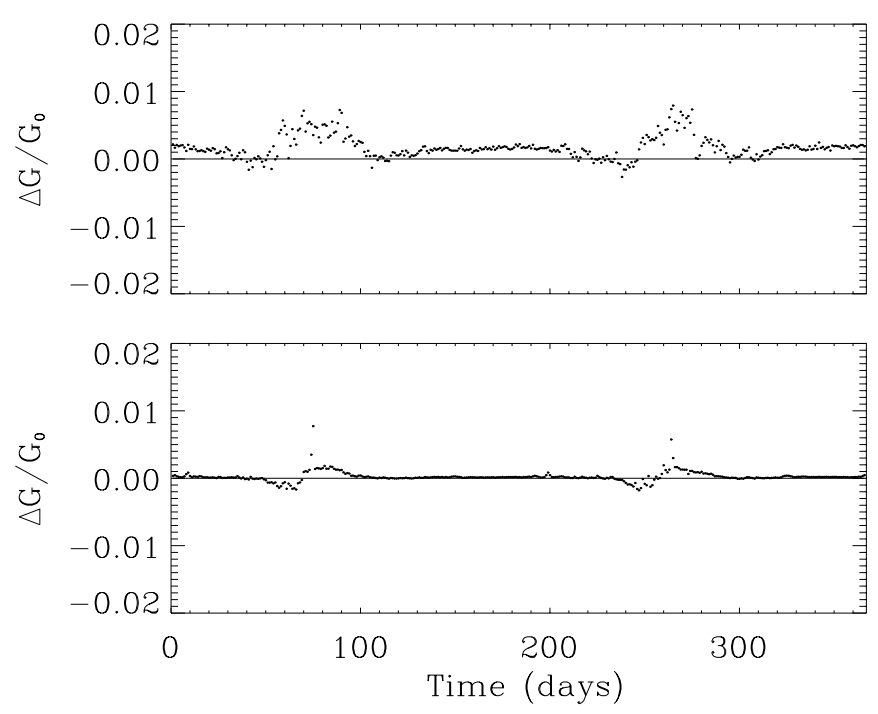

Fig. 13. Systematic error on $G$ calculated every 24 hours, with $\alpha=2.875$ at $30 \mathrm{GHz}$ (upper panel) and with $\alpha=0.75$ at $100 \mathrm{GHz}$ (lower panel).

The same anisotropy realizations, properly converted into antenna temperature, were used at both frequencies; we then obtain the observed sky:

$T^{\mathrm{obs}}=T^{\mathrm{dip}}+T^{\mathrm{gal}}+T^{\mathrm{cmb}}$

and the calibration sky:

$T^{\mathrm{cal}}=T^{\mathrm{dip}}+T^{\mathrm{gal}}$.

This is equivalent to neglecting CMB anisotropies in the calibration procedure: we assume no information at all on their presence.

Results on systematic errors are shown in the upper panels of Figs. 14 and 15. Note that the deviations are quite significant, exceeding $5 \%$ in some periods of the observations. The largest deviations are due to large scale CMB anisotropy structures, as one can anticipate based on the PLANCK scanning strategy.
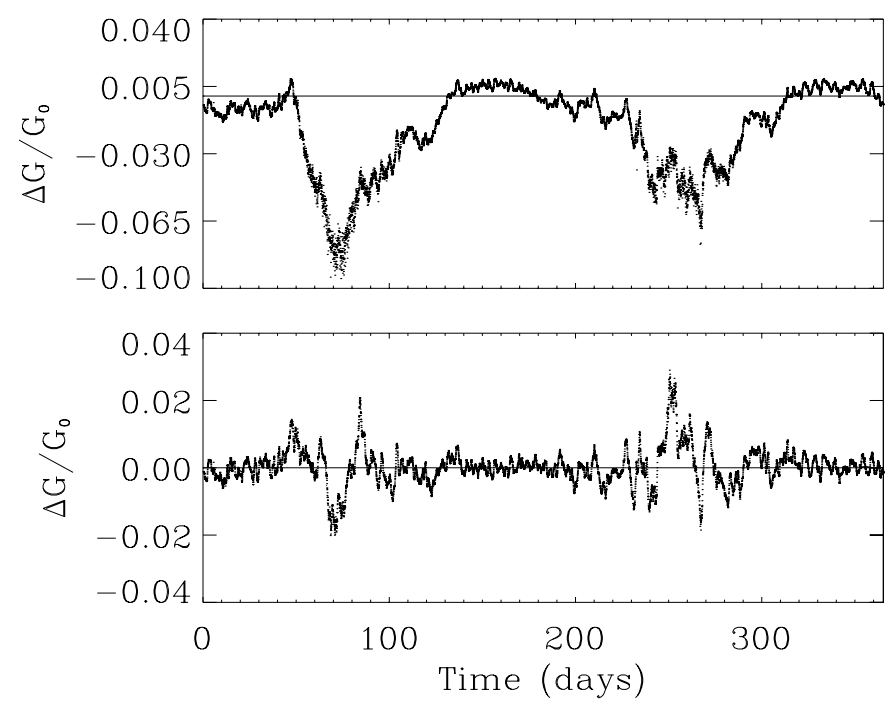

Fig. 14. $30 \mathrm{GHz}$. Upper panel: systematic error on calibration due to CMB anisotropies. Lower panel: systematic error calculated taking into account information we have on CMB amplitudes on large $\left(\theta \geq 7^{\circ}\right)$ scales.

In fact, if we consider the information on CMB amplitudes on large scales (such as the $C O B E$-DMR ones on $\theta \geq 7^{\circ}$ ) in the calibrator sky, and LFI-like angular resolution CMB map in the observed sky, so that

$$
\begin{aligned}
& T^{\mathrm{obs}}=T^{\mathrm{dip}}+T^{\mathrm{gal}}+T_{\mathrm{LFI}-\mathrm{FWHM}}^{\mathrm{cmb}} \\
& T^{\mathrm{cal}}=T^{\mathrm{dip}}+T^{\mathrm{gal}}+T_{\mathrm{COBE}-\mathrm{FWHM}}^{\mathrm{cmb}},
\end{aligned}
$$

only small scale structures continue to impact calibration, giving an error "randomly" distributed around zero (Figs. 14 and 15 , lower panels). To maintain the systematic error of calibration within $\sim 1 \%$, it is therefore necessary to use the information on the actual distribution of cold and hot spots in the CMB at large scales (e.g. $\gtrsim 7^{\circ}$ ).

\section{Conclusions}

The CMB dipole provides a nearly ideal source for calibration of extended maps of CMB anisotropy. For sensitive, full-sky surveys the modulation of the CMB dipole due to Earth rotation provides the most precisely known absolute calibration signal. However, preliminary absolute calibration on short-time scales needs to rely on the observed sky signal. Also, experiments mapping limited sky regions need to use the microwave sky as the calibration source. In this paper we have discussed an optimisation strategy using the sky emission as an absolute calibration source. In the absence of galactic foreground contributions, which are currently known with rather poor precision at millimeter wavelengths, high sensitivity, high resolution experiments could achieve calibrations accurate at $\leq 1 \%$ level on a time scale of 1 hour for nearly the entire mission. However, in practice, the presence of galactic diffuse emission introduces important systematic effects due to the uncertainties on their intensity and spatial structure. Cuts in galactic latitude can be used to mitigate this problem. However, residual systematic deviations in the recovery of the calibration gain are significant 

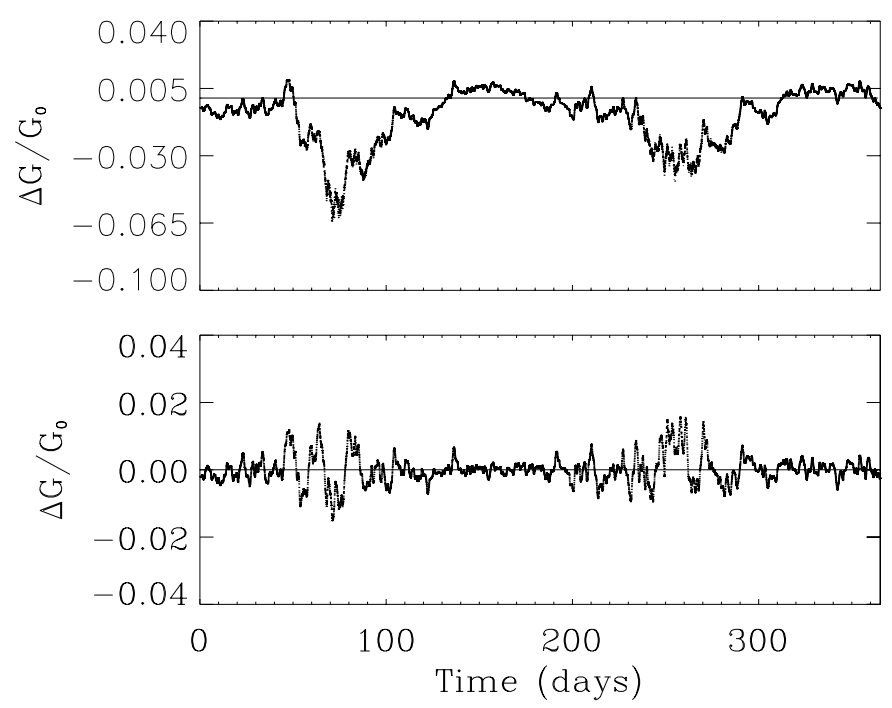

Fig. 15. $100 \mathrm{GHz}$. Upper panel: systematic error on calibration due to $\mathrm{CMB}$ anisotropies. Lower panel: systematic error calculated taking into account information we have on CMB amplitudes on large $\left(\theta \geq 7^{\circ}\right)$ scales.

(typically at the $10 \%$ level) due to the presence of high galactic latitude structures. We have shown that introducing a suitable weight function based on the estimated uncertainty in the total signal per pixel, it is possible to improve significantly the calibration accuracy with respect to methods simply involving cuts in galactic latitude. We applied this concept to the case of PLANCK-LFI, and produced quantitative estimates of the calibration accuracy with dedicated simulations. We have computed the optimal parameter $\alpha$ for the weight function, which, for a given experiment, depends on the frequency and time scale of calibration.

The results show that LFI with its nominal scanning strategy can reconstruct every hour the value of the gain parameter with $1 \%$ accuracy for $98 \%$ of the total time of the mission at $30 \mathrm{GHz}$ and for $99.5 \%$ at $100 \mathrm{GHz}$. If we consider an integration time of 1 day we obtain an accuracy $\leq 1 \%$ for $100 \%$ of the time at 30 and $100 \mathrm{GHz}$. Finally we have shown that the impact of the presence of the CMB anisotropies themselves is less than $1-2 \%$ for most of the time using the knowledge of their large-scale distribution from $C O B E$-DMR.

While these results have been obtained for the PLANCKLFI survey, the basic method can be applied to any precision measurements of the CMB on large sky areas. The recent measurements at mm wavelenghts by WMAP help to improve the a priori knowledge of the galactic signal. These new results can be readily incorporated in the proposed technique, leading to a more accurate determination of the optimum values of the $\alpha$ parameter; we will consider this in a forthcoming paper.

Future works include the analysis of the impact of instrumental systematics on the calibration procedure; we also need to study their behaviour with respect to the weight-function technique proposed in this paper.

\section{Appendix A: Calibration requirement}

The required precision on calibration depends on the goal precision in recovering the angular power spectrum coeficients $C_{\ell}$, typically a few $\%$ for precision experiments; this ensures cosmological parameters with comparable accuracy. We derive here a relation between a systematic error on the calibration - the $G$ factor - and the accuracy on the recovered power spectrum $C_{\ell}$, in the simple case where $G$ is constant over the whole map.

An error $\delta G$ on the gain factor induces an error on the measured $\Delta T$ :

$$
\begin{aligned}
\Delta T^{\prime} & =G^{\prime} \Delta V=G\left(1+\frac{\delta G}{G}\right) \Delta V \\
& =\left(1+\frac{\delta G}{G}\right) \Delta T
\end{aligned}
$$

then

$\Delta T^{\prime}-\Delta T=\frac{\delta G}{G} \Delta T$

In the first case the coefficients of the spherical decomposition of the $\Delta T$ function are

$a_{\ell m}=\int \frac{\Delta T}{T}(\theta, \phi) Y_{\ell m}(\theta, \phi) \mathrm{d} \Omega$

while in the second one

$a_{\ell m}^{\prime}=\int \frac{\Delta T^{\prime}}{T}(\theta, \phi) Y_{\ell m}(\theta, \phi) \mathrm{d} \Omega$.

Subtracting the previous two expression,

$$
\begin{aligned}
\delta a_{\ell m} & =\int \frac{\Delta T^{\prime}-\Delta T}{T}(\theta, \phi) Y_{\ell m}(\theta, \phi) \mathrm{d} \Omega \\
& =\int \frac{\delta G}{G} \frac{\Delta T}{T}(\theta, \phi) Y_{\ell m}(\theta, \phi) \mathrm{d} \Omega \\
& =\frac{\delta G}{G} a_{\ell m} .
\end{aligned}
$$

We recall the relation between $C_{\ell}$ and $a_{\ell m}$ coefficients:

$C_{\ell}=\frac{1}{2 \ell+1} \sum_{m=-\ell}^{\ell}\left|a_{\ell m}\right|^{2} ;$

differentiating

$$
\begin{aligned}
\delta C_{\ell} & =\frac{\partial C_{\ell}}{\partial a_{\ell m}} \delta a_{\ell m}=\frac{2}{2 \ell+1} \sum_{m=-\ell}^{\ell}\left|a_{\ell m}\right| \delta a_{\ell m} \\
& =\frac{2}{2 \ell+1} \frac{\delta G}{G} \sum_{m=-\ell}^{\ell}\left|a_{\ell m}\right|^{2}=2 \frac{\delta G}{G} C_{\ell} \\
\rightarrow \frac{\delta C_{\ell}}{C_{\ell}} & =2 \frac{\delta G}{G}
\end{aligned}
$$

In conclusion, in order to get $\delta C_{\ell} / C_{\ell} \simeq 1-2 \%$ one requires

$\frac{\delta G}{G} \leq 1 \%$. 


\section{References}

Bennett, C. L., Smoot, G. F., Janssen, M., et al. 1992, ApJ, 391, 466 Benoit, A., Ade, P., Amblard, A., et al. 2003, A\&A, 399, 19

Bersanelli, M., Muciaccia, P. F., Natoli, P., Vittorio, N., \& Mandolesi, N. 1997, A\&AS, 121, 393

Bersanelli, M., Maino, D., \& Mennella, A. 2002, La Rivista del Nuovo Cimento, 25(9), 1

Burigana, C., \& Paladini, R. 2003, Int. Rep. IASF-BO 370/2003, PLLFI-IASFbo-MA-004

De Bernardis, P., Ade, P., Bock, J. J., et al. 2000, Nature, 404, 955

Danese, L., Toffolatti, L., Franceschini, A., Bersanelli, M., \& Mandolesi, N. 1996, Astrophys. Lett. Commun., 33, 257

De Zotti, G., Toffolatti, L., Argüeso, F., et al. 1999, in 3 K Cosmology, AIP Conf. Proc., 476, 204

Draine, B. T., \& Lazarian, A. 1998, ApJ, 494, L19

Finkbeiner, D. P., Davis, M., \& Schlegel, D. J. 1999, ApJ, 542, 867

Fixsen, D., Cheng, E. S., Gales, J. M., et al. 1996, ApJ, 473, 576
Górski, K. M., Hivon, E., \& Wandelt, B. D. 1999, Proc. of the MPA/ESO Conference on Evolution of Large-Scale Structure: from Recombination to Garching, ed. A. J. Banday, R. K. Sheth, \& L. Da Costa, p. 37 [astro-ph/9812350]

Halverson, N. W., Leitch, E. M., Pryke, C., et al. 2002, ApJ, 568, 38 Hanany, S., Ade, P., Balbi, A., et al. 2000, ApJ, 545, L5

Haslam, C. G. T., Stoffel, H., Salter, C. J., \& Wilson, W. E. 1982, A\&AS, 47, 1

Jonas, J., Baart, E. E., \& Nicolson, G. D. 1998, MNRAS, 297, 997

Maino, D., Burigana, C., Maltoni, M., et al. 1999, A\&AS, 140, 383

Paladini, R., Burigana, C., Davies, R. D., et al. 2003, A\&A, 397, 213

Pearson, T. J., Mason, B. S., Readhead, A. C. S., et al. 2003, ApJ, 591, 556

Piat, M., Lagache, G., Bernard, J. P., Giard, M., \& Puget, J. L. 2002, A\&A, 393, 359

Reich, P., \& Reich, W. 1986, A\&AS, 63, 205

Schlegel, D. J., Finkbeiner, D. P., \& Davis, M. 1998, ApJ, 500, 525

Smoot, G. F., Bennett, C. L., Kogut, A., et al. 1992, ApJ, 396, L1 